\title{
Predictive Value of C-Reactive Protein for Major Postoperative Complications Following Off-Pump Coronary Artery Bypass Surgery Prospective and Observational Trial
}

\author{
Dae Hee Kim, MD; Jae Kwang Shim, MD, PhD*,\#; Seong Wook Hong, MD*; \\ Kwang Rae Cho, MD*; Seung Youn Kang, MD*; Young Lan Kwak, MD, PhD*,\#
}

\begin{abstract}
Background: To prospectively investigate the predictive value of the preoperative C-reactive protein (CRP) concentration for major postoperative complications following off-pump coronary artery bypass (OPCAB) surgery. Methods and Results: From January 2007 to December 2007, 185 consecutive patients scheduled for elective OPCAB surgery were allocated to a low-CRP group $(\mathrm{n}=137, \mathrm{CRP}<0.3 \mathrm{mg} / \mathrm{dl})$ and a high-CRP group $(\mathrm{n}=48, \mathrm{CRP}$ $\geq 0.3 \mathrm{mg} / \mathrm{dl}$ ). The incidence of major postoperative complications, defined as postoperative myocardial infarction, and 5 major morbidity endpoints including permanent stroke, renal dysfunction, any cardiac surgery reoperation, ventilation for more than $48 \mathrm{~h}$, and deep sternal wound infection were assessed and compared. Multivariate logistic regression was used to determine the predictors of major postoperative complications. Patients in the high-CRP group had a significantly higher overall incidence of major postoperative complications, particularly renal dysfunction. In the multivariate logistic regression model, adjusting all the significant univariate predictors, baseline CRP $>0.3 \mathrm{mg} / \mathrm{dl}$ and preoperative chronic renal failure (CRF) remained as significant independent predictors of major postoperative complications.

Conclusions: Elevated preoperative CRP level and/or preoperative CRF indicate increased risk of developing major postoperative complications, particularly acute postoperative renal dysfunction in patients undergoing OPCAB surgery. (Circ J 2009; 73: 872-877)
\end{abstract}

Key Words: C-reactive protein; Off-pump coronary artery bypass surgery; Postoperative morbidity

$\Delta$ ter cardiac surgery, the inflammatory reaction plays a pivotal role in the development of postoperative morbidity and mortality 1,2 Accordingly, the preoperative inflammatory status of the patient will significantly affect the degree of systemic inflammation and the postoperative outcome 3,4 Among the biomarkers of inflammatory status, C-reactive protein (CRP) is the most widely used5 Numerous studies have incontrovertibly demonstrated the strong predictive value of high-sensitivity CRP (hsCRP) for adverse cardiac events in patients undergoing percutaneous coronary intervention (PCI) and with myocardial infarction $(\mathrm{MI})^{6-10}$ As a result, CRP is rapidly evolving into a stronger risk predictor than other novel risk factors in the setting of coronary ischemia and unstable angina. However, its predictive role and clinical application in patients undergoing

(Received October 26, 2008; revised manuscript received November 22, 2008; accepted December 11, 2008; released online March 13, 2009)

Department of Anesthesiology and Pain Medicine, Ajou University College of Medicine, Suwon, *Department of Anesthesiology and Pain Medicine and "Anesthesia and Pain Research Institute, Yonsei University College of Medicine, Seoul, South Korea

Mailing address: Young Lan Kwak, MD, PhD, Department of Anesthesiology and Pain Medicine and Anesthesia and Pain Research Institute, Yonsei University College of Medicine, 134 Shinchon-Dong, Seodaemun-Ku, Seoul 120-752, South Korea. E-mail: ylkwak@yuhs. ac

All rights are reserved to the Japanese Circulation Society. For permissions, please e-mail: cj@j-circ.or.jp coronary artery bypass graft surgery $(\mathrm{CABG})$ remains controversial? $3,4,11-14$ Moreover, most of the studies are pertinent to on-pump CABG, and no comprehensive data exist for its predictive value in off-pump coronary artery bypass surgery (OPCAB).

\section{Editorial p818}

The regional warm, instead of the global cold, ischemiareperfusion injury imposed on the myocardium and the avoidance of cardiopulmonary bypass (CPB) in $\mathrm{OPCAB}$ would confer a different impact of the degree of inflammatory reaction and, consequently, the postoperative outcome $!^{5}$ Thus, in terms of the degree of the inflammatory response and myocardial ischemia, OPCAB would stand between on-pump CABG and PCI, and a more distinct role of the preoperative CRP level is expected in OPCAB patients compared with on-pump CABG. We, therefore, designed a prospective and observational trial to investigate the predictive value of the preoperative hsCRP level for major postoperative complications following $\mathrm{OPCAB}$ during the postoperative hospitalization period.

\footnotetext{
Methods

Patients

A total of 185 consecutive patients scheduled for elective OPCAB from January 2007 to December 2007 were included. Patients were excluded because of emergency or
} 
Table 1. Baseline and Intraoperative Characteristics of the Patients

\begin{tabular}{lccc}
\hline & Low-CRP $(\mathrm{n}=137)$ & High-CRP $(\mathrm{n}=48)$ & P value \\
\hline Age (years) & $64.0 \pm 8.7$ & $66.9 \pm 7.3$ & 0.037 \\
Sex $(\mathrm{M} / \mathrm{F})$ & $98 / 39$ & $36 / 12$ & 0.644 \\
Body surface area $\left(\mathrm{m}^{2}\right)$ & $1.71 \pm 0.17$ & $1.70 \pm 0.16$ & 0.734 \\
History & & & 0.367 \\
$\quad$ Hypertension & $98(72)$ & $31(65)$ & 0.754 \\
Diabetes mellitus & $52(40)$ & $17(35)$ & 0.542 \\
CRF & $10(7)$ & $5(10)$ & 0.304 \\
CVA & $7(5)$ & $5(10)$ & 0.412 \\
PAD & $15(11)$ & $3(6)$ & 0.715 \\
Previous MI & $39(28)$ & $15(31)$ & 0.320 \\
Creatinine (mg/dl) & $1.1 \pm 0.5$ & $1.2 \pm 1.1$ & 0.573 \\
NYHA class $\geq 3$ & $48(35)$ & $19(40)$ & 0.549 \\
Preoperative medications & & & 0.818 \\
Nitrate & $56(41)$ & $22(46)$ & 0.086 \\
$\beta$-blockers & $105(77)$ & $36(75)$ & 0.578 \\
ACEI & $24(18)$ & $14(29)$ & 0.406 \\
ARB & $37(27)$ & $11(23)$ & 0.141 \\
CCB & $78(57)$ & $24(50)$ & 0.107 \\
Aspirin & $101(74)$ & $30(63)$ & 0.789 \\
Clopidogrel & $92(67)$ & $26(54)$ & 0.500 \\
Statins & $97(71)$ & $33(69)$ & 0.679 \\
LVEF $(\%)$ & $59 \pm 12$ & $57 \pm 12$ & 0.570 \\
Coronary artery disease & & & 0.609 \\
Left main & $6(4)$ & $1(2)$ & 0.273 \\
3-vessel disease & $109(80)$ & $40(83)$ & $3.3 \pm 0.7$ \\
No. of anastomoses & $3.3 \pm 0.7$ & $259 \pm 69$ & \\
Duration of operation (min) & $272 \pm 72$ & & \\
\hline
\end{tabular}

Values are mean \pm SD or number of patients $(\%)$.

CRP, C-reactive protein; CRF, chronic renal failure; CVA, cerebrovascular accident; PAD, peripheral artery obstructive disease; MI, myocardial infarction; NYHA class, New York Heart Association functional class; ACEI, angiotensin-converting enzyme inhibitor; ARB, angiotensin-receptor blocker; CCB, calcium-channel blocker; LVEF, left ventricular ejection fraction.

urgent surgery, recent MI within the 1 month of the time of enrolment, age under 20 years, known malignancy, active infection, autoimmune disease, or chronic anti-inflammatory therapy other than low-dose aspirin. The study was conducted according to the principles of the Declaration of Helsinki, and written informed consent was given by all patients. The institutional ethics committee approved the study.

The following preoperative data were prospectively collected: age, sex, body surface area, serum creatinine level, left ventricular ejection fraction (LVEF) and medications. Assessment of comorbidities included history of hypertension, diabetes mellitus under medical treatment, neurologic events, chronic renal failure (CRF defined as estimated glomerular filtration rate $<60 \mathrm{ml} \cdot \mathrm{min}^{-1} \cdot 1.73 \mathrm{~m}^{-2}$ ), vascular disease, and documented history of prior MI.

\section{Anesthetic Management and Operative Technique}

All patients received standardized anesthetic management. In the operating room, leads II and V5 of the ECG and arterial blood pressure were continuously monitored. A thermodilution pulmonary artery catheter was inserted for continuous monitoring of cardiac output and mixed venous oxygen saturation. Anesthesia was induced with intravenous midazolam $(0.03-0.07 \mathrm{mg} / \mathrm{kg})$, sufentanil $(1.5-3.0 \mu \mathrm{g} / \mathrm{kg})$ and rocuronium bromide $50 \mathrm{mg}$, and maintained with sevoflurane $(0.8-1.5 \mathrm{vol} \%)$ and continuous infusion of sufentanil $\left(0.5-1.5 \mu \mathrm{g} \cdot \mathrm{kg}^{-1} \cdot \mathrm{h}^{-1}\right)$.

All surgical procedures were performed by the same surgeon through a median sternotomy. To avoid instrumentation of the ascending aorta, a "no-touch" technique 16 was used whenever possible. With this technique, revascularization was carried out using 1 or both internal thoracic arteries connected with an additional graft (mainly the radial artery, and/or saphenous vein) in a Y configuration. To expose and stabilize the target coronary vessel, a deep pericardial suture and suction stabilizer $\left(\right.$ Rosta $^{\circledR} 2.0$, Yoorim Corporation, Chungbuck, Korea) was used. During distal anastomosis, mean systemic arterial pressure was maintained within 70$80 \mathrm{mmHg}$ either with a $10-20^{\circ}$ Trendelenburg's position and/or norepinephrine infusion. The number of distal anastomoses and operation time were recorded. All patients were transferred to the intensive care unit (ICU) after operation.

\section{Biochemistry}

hsCRP was measured by the high-sensitivity nephelometric method (Dade Behring Marburg GMBH, Marburg, Germany) on the morning of the day before surgery, and on the morning of the $1^{\text {st }}, 2^{\text {nd }}$, and $3^{\text {rd }}$ postoperative days (POD 1, 2 and 3). The lower detection limit of this method was $<0.015 \mathrm{mg} / \mathrm{dl}$. Creatine kinase-myocardial band (CK-MB) and troponin-T (Tn-T) levels were also measured at the same time points. According to the baseline level of hsCRP, patients were allocated to a low-CRP group, which included patients with hsCRP level $<0.3 \mathrm{mg} / \mathrm{dl}(\mathrm{n}=137)$, and a highCRP group, which included patients with hsCRP level $\geq 0.3 \mathrm{mg} / \mathrm{dl}$ ( $\mathrm{n}=48)$. The cut-off point of $0.3 \mathrm{mg} / \mathrm{dl}$ was based on the American Heart Association and Centers for Disease Control and Prevention scientific statement suggesting that levels greater than $0.3 \mathrm{mg} / \mathrm{dl}$ be considered high ${ }^{7}$

\section{Postoperative Evaluation}

The postoperative clinical course of all patients, including the duration of mechanical ventilation, re-intubation rate, chest tube drainage for $72 \mathrm{~h}$, transfusion requirement, use of inotropic agents or vasopressors, new onset of atrial fibril- 
Table 2. Postoperative Outcome Variables

\begin{tabular}{lccc}
\hline & Low-CRP $(\mathrm{n}=137)$ & High-CRP $(\mathrm{n}=48)$ & P value \\
\hline Duration of ventilatory care $(\mathrm{h})$ & $15.8 \pm 22.6$ & $15.6 \pm 9.5$ & 0.950 \\
Re-intubation & $5(4)$ & $1(2)$ & 1.000 \\
Chest tube drainage (ml) & $1,538 \pm 584$ & $1,509 \pm 636$ & 0.772 \\
Transfusion & $56(41)$ & $16(33)$ & 0.356 \\
Inotropic agent support & $17(12)$ & $7(15)$ & 0.700 \\
Vasopressor support & $18(13)$ & $4(8)$ & 0.376 \\
Atrial fibrillation & $23(17)$ & $12(25)$ & 0.211 \\
Major postoperative complications & $14(10)$ & $14(29)$ & 0.002 \\
Postoperative MI & $6(4)$ & $5(10)$ & 0.160 \\
5-major STS morbidity endpoints & $9(7)$ & $10(21)$ & 0.010 \\
$\quad$ Stroke & $1(1)$ & 0 & 1.000 \\
Cardiac reoperation & $1(1)$ & $2(4)$ & 0.166 \\
Deep sternal infection & $1(1)$ & 0 & 1.000 \\
Renal dysfunction & $4(3)$ & $7(15)$ & 0.008 \\
Ventilator care $>48 \mathrm{~h}$ & $4(3)$ & $1(2)$ & 1.000 \\
Length of ICU stay (days) & $2.3 \pm 1.2$ & $2.5 \pm 1.7$ & 0.427 \\
Length of postoperative hospital stay (days) & $10.4 \pm 9.9$ & $11.4 \pm 8.2$ & 0.563 \\
Mortality & $2(1)$ & 0 & 1.000 \\
\hline
\end{tabular}

Values are mean \pm SD or number of patients (\%).

Inotropic agents, dobutamine or milrinone infusion; Vasopressors, norepinephrine $>0.2 \mu \mathrm{g} \cdot \mathrm{kg}^{-1} \cdot \mathrm{min}^{-1}$ or vasopressin infusion; STS, the Society of Thoracic Surgeons; ICU, intensive care unit. Other abbreviations see in Table 1.

lation, and postoperative MI and the 5 major morbidity endpoints were recorded until hospital discharge. The length of stay in the ICU and the postoperative hospitalization, as well as the in-hospital mortality, were also recorded. MI was defined as the occurrence of an increase in CK-MB $\geq 25 \mathrm{ng} / \mathrm{dl}$ (5-fold above the upper normal limit) and/or development of new pathologic Q wave on electrocardiography. The 5 major morbidity endpoints were defined as permanent stroke, renal dysfunction, any cardiac surgery reoperation, ventilation $>48 \mathrm{~h}$, and deep sternal wound infection ${ }^{18}$ For clarification, renal dysfunction was defined as acute postoperative renal insufficiency resulting in 1 or more of the following: (1) increase of serum creatinine to $>2.0 \mathrm{mg} / \mathrm{dl},(2) \geq 50 \%$ increase in creatinine over baseline preoperative value, or (3) new requirement for dialysis. Inhospital mortality was defined as death within the same hospital admission regardless of cause.

\section{Statistical Analysis}

Data are expressed as mean \pm standard deviation or number of patients (\%) and were analyzed with SPSS (version 13, SPSS Inc, Chicago, IL, USA). The primary objective of this study was to compare the overall incidence of the major postoperative complications defined as postoperative MI and 5 major morbidity endpoints between groups, and to analyze the predictive value of hsCRP. To compare the low- and high-CRP groups, chi-square or Fisher's exact test for categorical variables and unpaired t-test or Mann-Whitney $\mathrm{U}$ test for continuous variables were used as appropriate. To determine the predictive value of hsCRP and other preoperative variables for major postoperative complications, multivariate analysis was used with logistic regression using a forward stepwise selection method. Preoperative variables of $\mathrm{P}<0.2$ in the univariate analysis were included in the regression model. A P-value $<0.05$ was considered statistically significant.

\section{Results}

\section{Patients}

OPCAB was successfully performed in all patients without emergency conversion to on-pump CABG. Patients' characteristics and operative data, including the number of grafts performed, were similar between the groups except for age (Table 1).

Postoperative outcome variables are shown in Table 2. The overall incidence of the predefined major postoperative complications was significantly higher in the high-CRP group. Other postoperative outcome variables were comparable between the groups. Among the predefined major postoperative complications, the overall incidence of the 5 major morbidity endpoints was significantly higher in the high-CRP group, although the incidence of postoperative MI was also higher in the high-CRP group without statistical significance $(10 \%$ vs $4 \%, \mathrm{P}=0.160)$. When comparing each parameter constituting the 5 major morbidity endpoints, only the incidence of renal dysfunction was significantly higher in the high-CRP group. Of the patients who developed renal dysfunction, 1 in the high-CRP group required hemodialysis. The creatinine levels returned to preoperative values in all patients before hospital discharge. Both the length of ICU stay and postoperative hospitalization were similar between the groups. The in-hospital mortality rate was also without statistical significance, and the cause of death of 2 patients in the low-CRP group was graft failure and subsequent cardiac arrest, respectively.

\section{Biomarkers}

Although the baseline hsCRP levels were essentially different, postoperative CRP showed no differences, peaking on POD 2 in both groups. The white blood cell (WBC) count was significantly higher in the high-CRP group on POD 2. There were no significant differences in the CK-MB and Tn-T levels at baseline or on PODs 1-3 (Table 3).

\section{Multivariate Logistic Regression}

To determine the risk factors for major postoperative complications, suspected univariate predictors (all perioperative variables shown in Table 1 and baseline CRP, WBC count, CK-MB, and Tn-T) were assessed, and significant univariate predictors included lower LVEF, history of MI, CRF, cerebrovascular accident, and baseline hsCRP 
Table 3. Perioperative Values of Cardiac and Inflammatory Biomarkers

\begin{tabular}{|c|c|c|c|}
\hline & Low-CRP (n=137) & High-CRP $(n=48)$ & $P$ value \\
\hline \multicolumn{4}{|l|}{ CRP (mg/dl) } \\
\hline Preoperative & $0.097 \pm 0.074$ & $1.146 \pm 1.048$ & $<0.001$ \\
\hline POD 1 & $6.840 \pm 2.641$ & $7.572 \pm 2.694$ & 0.106 \\
\hline POD 2 & $16.184 \pm 8.460$ & $16.481 \pm 6.437$ & 0.844 \\
\hline POD 3 & $12.060 \pm 4.941$ & $13.717 \pm 5.499$ & 0.221 \\
\hline \multicolumn{4}{|l|}{$\operatorname{WBC}\left(10^{3} / \mu 1\right)$} \\
\hline Preoperative & $7.1 \pm 1.8$ & $7.7 \pm 2.4$ & 0.100 \\
\hline POD 1 & $11.1 \pm 2.8$ & $11.7 \pm 3.7$ & 0.352 \\
\hline POD 2 & $10.6 \pm 3.5$ & $12.1 \pm 4.2$ & 0.020 \\
\hline POD 3 & $9.1 \pm 2.9$ & $9.7 \pm 2.7$ & 0.205 \\
\hline \multicolumn{4}{|l|}{ CK-MB (ng/ml) } \\
\hline Preoperative & $3.33 \pm 1.60$ & $4.44 \pm 6.39$ & 0.306 \\
\hline POD 1 & $9.76 \pm 9.45$ & $11.12 \pm 8.94$ & 0.388 \\
\hline POD 2 & $7.76 \pm 5.75$ & $7.05 \pm 4.15$ & 0.474 \\
\hline POD 3 & $4.43 \pm 2.25$ & $4.52 \pm 2.16$ & 0.882 \\
\hline \multicolumn{4}{|l|}{ Tn-T (ng/ml) } \\
\hline Preoperative & $0.017 \pm 0.074$ & $0.097 \pm 0.324$ & 0.151 \\
\hline POD 1 & $0.140 \pm 0.161$ & $0.441 \pm 1.564$ & 0.209 \\
\hline POD 2 & $0.160 \pm 0.302$ & $0.467 \pm 1.521$ & 0.223 \\
\hline POD 3 & $0.171 \pm 0.335$ & $0.214 \pm 0.349$ & 0.651 \\
\hline
\end{tabular}

Values are mean \pm SD.

POD, postoperative day; WBC, white blood cell count; CK-MB, creatine kinase-MB; TnT, troponin T. Other abbreviation see in Table 1.

Table 4. Univariate and Multivariate Preoperative Predictors of Major Postoperative Complications

\begin{tabular}{lccc}
\hline Predictors & $\begin{array}{c}\text { Univariate } \\
\text { P value }\end{array}$ & $\begin{array}{c}\text { Multivariate } \\
\text { OR (95\%CI) }\end{array}$ & P value \\
\hline LVEF & 0.156 & & \\
History of previous MI & 0.029 & & \\
Preoperative CRF & 0.002 & $6.163(1.913-19.855)$ & 0.002 \\
Preoperative CVA & 0.088 & $3.833(1.602-9.172)$ & 0.003 \\
Preoperative CRP $>0.3 \mathrm{mg} / \mathrm{dl}$ & 0.002 & 3 \\
\hline
\end{tabular}

OR, odds ratio; CI, confidence interval. Other abbreviations see in Table 1.

$>0.3 \mathrm{mg} / \mathrm{dl}$. In the multivariate logistic regression model, adjusting all the significant univariate predictors, preoperative CRF and baseline hsCRP $>0.3 \mathrm{mg} / \mathrm{dl}$ remained significant independent predictors of postoperative major complications (Table 4).

When the predictive value of the same risk factors for the development of postoperative renal dysfunction alone were analyzed in a multivariate logistic regression model, preoperative CRF (odds ratio (OR) [95\% confidence interval (CI)], 10.173 [2.012-51.440]) and elevated preoperative CRP level (OR [95\%CI], 4.883 [1.168-20.410]) were also identified as significant independent predictors with higher OR.

\section{Discussion}

In the current study addressing the predictive value of the CRP level for a patient's short-term prognosis following OPCAB, we found that the overall incidence of major postoperative complications was significantly higher in the high-CRP group. Among the postoperative complications, the incidence of renal dysfunction was significantly higher, and the incidences of MI and cardiac reoperation showed a trend toward being higher in the high-CRP group. Furthermore, high preoperative hsCRP level and history of CRF were independent risk factors for the development of major postoperative complications, especially acute postoperative renal dysfunction.
Identification of factors related to an increased risk of postoperative complications allows accurate risk stratification, and provides the rationale for optimizing certain medical conditions of the patient before the surgery, if possible. During the past decade, CRP has emerged as a significant predictor of cardiovascular events in both healthy subjects and patients with coronary artery disease, with consistent clinical evidence6-10 Furthermore, the CRP level has been shown to be an independent predictor of future cardiovascular events, adding prognostic information to the Framingham Risk Score, to lipid screening, and to the metabolic syndrome 19 Thus, in the area of primary prevention, the usefulness of the criterion encompassing the CRP modified ischemic heart disease risk score for global risk prediction is being increasingly recognized? 20

Cardiac surgery, particularly if requiring $\mathrm{CPB}$, invariably produces a broad and intense systemic inflammatory response to a different extent among individual patients? ${ }^{21}$ This systemic inflammatory response, together with microembolization, is being held responsible for the development of most of the morbidities following cardiac surgery, and not surprisingly, the preoperative inflammatory status of the patient significantly affects the degree of systemic inflammatory response during cardiac surgery. However, unlike in the setting of coronary artery disease or PCI, the reported predictive value of the preoperative CRP level for postoperative outcome in on-pump CABG has been controversial?,4,11-14 Strong associations between an elevated hsCRP 
level with an increased overall incidence of infection and sepsis, short- and long-term postoperative mortality, and early graft occlusion has been demonstrated by previous studies, $32,14,22$ In contrast, a preoperative level of CRP as high as $>5 \mathrm{mg} / \mathrm{L}$ could not predict in-hospital postoperative complications following primary isolated on-pump CABG4 In a prospective study, Tn-T but not CRP was identified as a predictive variable for short-term prognosis following onpump CABG and those authors suggested that myocardial ischemia plays the main role in on-pump CABG, in contrast with PCI where inflammation is the cornerstone? 3

OPCAB is associated with a reduced inflammatory reaction and myocardial injury because it avoids the use of CPB and does not impose a global cold myocardial ischemia, but there is still a significant acute inflammatory response as a result of surgical trauma and warm regional myocardial ischemia ${ }^{15}$ In terms of the extent of both the inflammatory reaction and myocardial ischemia, we consider that the effect of OPCAB is between that of on-pump CABG and PCI. Therefore, the prognostic importance of CRP for the patient's outcome would be more definite in OPCAB than in on-pump CABG, although evidence to prove this hypothesis is lacking to date.

As our results indicate, a high preoperative hsCRP level was associated with frequent development of major postoperative complications, especially renal dysfunction. In addition, a high hsCRP, together with preoperative CRF, was identified as an independent predictor for major postoperative complications in our multivariate analysis. Postoperative MI and the 5 major morbidity endpoints are well known as affecting patients' quality of life and may often pose a serious threat to a patient's long-term survival and overall wellbeing after the operation! 18

Of interest is that when comparing each parameter constituting the major postoperative complications, only the incidence of renal dysfunction was significantly higher in the high-CRP group. Moreover, together with preoperative $\mathrm{CRF}$, an elevated preoperative CRP level was an independent risk factor for postoperative renal dysfunction, in particular, on our multivariate logistic regression analysis. Preexisting renal impairment has been consistently identified as a major predictor for postoperative renal failure in OPCAB ${ }^{24,25}$ However, although not surprising, this strong association of an elevated preoperative hsCRP level with the development of postoperative renal dysfunction following OPCAB is a novel finding of this study. Renal dysfunction after cardiac surgery is well known as having a significant influence on postoperative morbidity and mortality, and although the causes of renal dysfunction after cardiac surgery are multifactorial, the inflammatory response is 1 of the important contributors? ${ }^{26}$ In support of the association between elevated preoperative hsCRP level and renal dysfunction, the preoperative inflammatory status as measured by the hsCRP level was closely associated with the postoperative inflammatory reaction and has been shown to magnify the effects of intraoperative inflammatory stressors ${ }^{13,14}$ Moreover, renal filtration contributes to the clearance of many inflammatory cytokines and thus postoperative renal dysfunction per se may also influence the course and magnitude of the inflammatory response, thereby effecting other major complications, including aggravation of the already present renal dysfunction. ${ }^{27}$ Based on these perspectives and our results, therapeutic efforts should be made to attenuate the inflammatory response and/or to protect the kidney in patients with an elevated preoperative hsCRP level present- ing for OPCAB. For that purpose, statin therapy could be a promising therapeutic strategy, as those drugs have been shown to reduce the levels of certain inflammatory markers, such as hsCRP and IL-6, and to dramatically reduce morbidity and mortality in patients with established cardiovascular disease ${ }^{2-30}$ However, the efficacy of statins in reducing major postoperative complications in patients undergoing $\mathrm{OPCAB}$ remains to be proven.

\section{Study Limitations}

hsCRP is a well-established marker of inflammation, and despite the preoperative difference in the hsCRP levels and the theory of accentuated inflammatory response in patients with increased baseline inflammatory status, the postoperative hsCRP levels were similar between the groups in this study. Therefore, the suggestion that an increased inflammatory response resulted in the increased incidence of major postoperative complications in the high-CRP group may not seem reasonable. However, hsCRP is only 1 of many inflammatory markers, and thus judging the patient's inflammatory response solely by the serum level of CRP may not be acceptable. The finding that leukocytosis was more pronounced in the high-CRP group on POD 2 also implies that there was an increased inflammatory response in the high-CRP group. Moreover, the fact that a preoperative elevated hsCRP level was an independent predictor of major postoperative complications, despite the similarity in the postoperative hsCRP levels, further strengthens the predictive value of hsCRP.

In conclusion, we found a strong association between an elevated preoperative hsCRP level and the occurrence of major postoperative complications following OPCAB. Furthermore, together with preoperative $\mathrm{CRF}$, a preoperative level of CRP $>0.3 \mathrm{mg} / \mathrm{dl}$ was an independent risk factor for the development of postoperative major complications, particularly renal dysfunction.

\section{Acknowledgment}

We thank Dr Kyung-Jong Yoo (Department of Thoracic and Cardiovascular Surgery, Yonsei University College of Medicine) who performed the OPCAB in this study.

\section{References}

1. Hennein HA, Ebba H, Rodriguez JL, Merrick SH, Keith FM, Bronstein $\mathrm{MH}$, et al. Relationship of the proinflammatory cytokines to myocardial ischemia and dysfunction after uncomplicated coronary revascularization. J Thorac Cardiovasc Surg 1994; 108: 626-635.

2. Hill GE. Cardiopulmonary bypass-induced inflammation: Is it important? J Cardiothorac Vasc Anesth 1998; 12: 21-25.

3. Biancari F, Lahtinen J, Lepojärvi $S$, Rainio $P$, Salmela E, Pokela R, et al. Preoperative C-reactive protein and outcome after coronary artery bypass surgery. Ann Thorac Surg 2003; 76: 2007-2012.

4. Gaudino M, Nasso G, Andreotti F, Minniti G, Iacoviello L, Donati M, et al. Preoperative C-reactive protein level and outcome following coronary surgery. Eur J Cardiothorac Surg 2002; 22: 521-526.

5. Ablij H, Meinders A. C-reactive protein: History and revival. Eur $J$ Intern Med 2002; 13: 412-422.

6. Goldberg A, Gruberg L, Roguin A, Petcherski S, Rimer D, Markiewicz W, et al. Preprocedural C-reactive protein levels predict myocardial necrosis after successful coronary stenting in patients with stable angina. Am Heart J 2006; 151: 1265-1270.

7. Rahel BM, Visseren FL, Suttorp MJ, Plokker TH, Kelder JC, de Jongh BM, et al. Preprocedural serum levels of acute-phase reactants and prognosis after percutaneous coronary intervention. Cardiovasc Res 2003; 60: 136-140.

8. Gach O, Legrand V, Biessaux Y, Chapelle JP, Vanbelle S, Pierard LA. Long-term prognostic significance of high-sensitivity C-reactive protein before and after coronary angioplasty in patients with stable angina pectoris. Am J Cardiol 2007; 99: 31-35. 
9. Kim H, Yang DH, Park Y, Han J, Lee H, Kang H, et al. Incremental prognostic value of $\mathrm{C}$-reactive protein and $\mathrm{N}$-terminal proB-type natriuretic peptide in acute coronary syndrome. Circ J 2006; 70: $1379-1384$

10. Hong YJ, Jeong MH, Lim SY, Lee SR, Kim KH, Sohn IS, et al. Elevated preprocedural high-sensitivity C-reactive protein levels are associated with neointimal hyperplasia and restenosis development after successful coronary artery stenting. Circ J 2005; 69: 14771483.

11. Palmerini T, Marzocchi A, Marrozzini C, Reggiani LB, Savini C, Marinelli G, et al. Preoperative C-reactive protein levels predict 9month mortality after coronary artery bypass grafting surgery for the treatment of left main coronary artery stenosis. Eur J Cardiothorac Surg 2007; 31: 685-690.

12. Kangasniemi OP, Biancari F, Luukkonen J, Vuorisalo S, Satta J, Pokela R, et al. Preoperative C-reactive protein is predictive of longterm outcome after coronary artery bypass surgery. Eur J Cardiothorac Surg 2006; 29: 983-985.

13. Boeken U, Feindt P, Zimmermann N, Kalweit G, Petzold T, Gams E. Increased preoperative $\mathrm{C}$-reactive protein (CRP)-values without signs of an infection and complicated course after cardiopulmonary bypass (CPB)-operations. Eur J Cardiothorac Surg 1998; 13: 541-545.

14. Cappabianca G, Paparella D, Visicchio G, Capone G, Lionetti G, Numis F, et al. Preoperative C-reactive protein predicts mid-term outcome after cardiac surgery. Ann Thorac Surg 2006; 82: 2170 2178.

15. Raja SG, Berg GA. Impact of off-pump coronary artery bypass surgery on systemic inflammation: Current best available evidence. J Card Surg 2007; 22: 445-455.

16. Leacche M, Carrier M, Bouchard D, Pellerin M, Perrault LP, Pagá P, et al. Improving neurologic outcome in off-pump surgery: The "no touch" technique. Heart Surg Forum 2003; 6: 169-175.

17. Pearson TA, Mensah GA, Alexander RW, Anderson JL, Cannon RO 3rd, Criqui M, et al. Markers of inflammation and cardiovascular disease: Application to clinical and public health practice: A statement for healthcare professionals from the Centers for Disease Control and Prevention and the American Heart Association. Circulation 2003; 107: 499-511.

18. Shroyer AL, Coombs LP, Peterson ED, Eiken MC, DeLong ER, Chen A, et al. The Society of Thoracic Surgeons: 30-day operative mortality and morbidity risk models. Ann Thorac Surg 2003; 75: $1856-1864$

19. Ridker PM. Clinical application of C-reactive protein for cardiovas- cular disease detection and prevention. Circulation 2003; 107: $363-$ 369.

20. Ridker PM, Wilson PW, Grundy SM. Should C-reactive protein be added to metabolic syndrome and to assessment of global cardiovascular risk? Circulation 2004; 109: 2818-2825.

21. Laffey JG, Boylan JF, Cheng DC. The systemic inflammatory response to cardiac surgery: Implications for the anesthesiologist. Anesthesiology 2002; 97: 215-252.

22. Hedman A, Larsson PT, Alam M, Wallen NH, Nordlander R, Samad BA. CRP, IL-6 and endothelin-1 levels in patients undergoing coronary artery bypass grafting: Do preoperative inflammatory parameters predict early graft occlusion and late cardiovascular events? Int $J$ Cardiol 2007; 120: 108-114.

23. Pascual DA, Arribas JM, Tornel PL, Marín F, Oliver C, Ahumada M, et al. Preoperative statin therapy and troponin T predict early complications of coronary artery surgery. Ann Thorac Surg 2006; 81: $78-$ 84.

24. Di Mauro M, Gagliardi M, Iacò AL, Contini M, Bivona A, Bosco P, et al. Does off-pump coronary surgery reduce postoperative acute renal failure?: The importance of preoperative renal function. Ann Thorac Surg 2007; 84: 1496-1502.

25. Chukwuemeka A, Weisel A, Maganti M, Nette AF, Wijeysundera DN, Beattie WS, et al. Renal dysfunction in high-risk patients after on-pump and off-pump coronary artery bypass surgery: A propensity score analysis. Ann Thorac Surg 2005; 80: 2148-2153.

26. Pontes JC, da Silva GV, Benfatti RA, Machado NP, Pontelli R, Pontes ER. Risk factors for the development of acute renal failure following on-pump coronary artery bypass grafting. Rev Bras Cir Cardiovasc 2007; 22: 484-490

27. Vanholder R, Argilés A, Baurmeister U, Brunet P, Clark W, Cohen $\mathrm{G}$, et al. Uremic toxicity: Present state of the art. Int J Artif Organs 2001; 24: 695-725.

28. Ridker PM, Cannon CP, Morrow D, Rifai N, Rose LM, McCabe CH, et al. C-reactive protein levels and outcomes after statin therapy. $N$ Engl J Med 2005; 352: 20-28.

29. Nissen SE, Tuzcu EM, Schoenhagen P, Crowe T, Sasiela WJ, Tsai J, et al. Statin therapy, LDL cholesterol, C-reactive protein, and coronary artery disease. $N$ Engl J Med 2005; 352: 29-38.

30. Pasceri V, Patti G, Nusca A, Pristipino C, Richichi G, Di Sciascio G. Randomized trial of atorvastatin for reduction of myocardial damage during coronary intervention: Results from the ARMYDA (Atorvastatin for Reduction of MYocardial Damage during Angioplasty) study. Circulation 2004; 110: 674-678. 